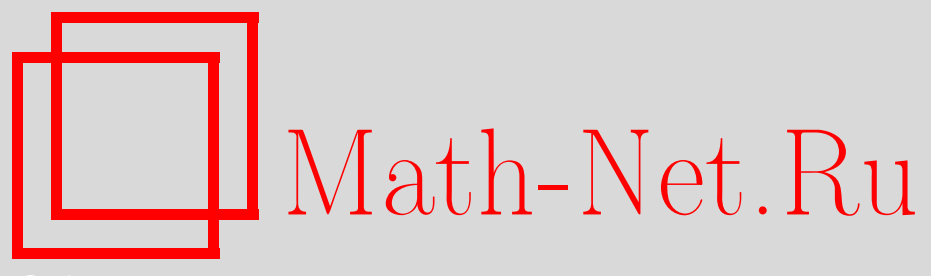

И. В. Кривченков, Развитие метода эйконала в нелинейной электродинамике на основе принципа геометризации, ТМФ, 2007, том 150, номер 1, 112-117

DOI: https://doi.org/10.4213/tmf5968

Использование Общероссийского математического портала Math-Net.Ru подразумевает, что вы прочитали и согласны с пользовательским соглашением http: //www. mathnet.ru/rus/agreement

Параметры загрузки:

IP : 54.157 .27 .8

26 апреля 2023 г., 15:40:56

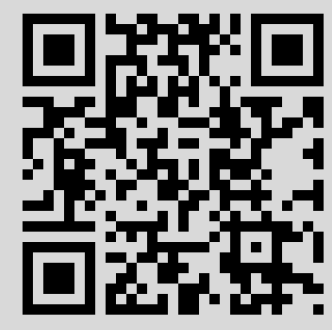




\title{
РАЗВИТИЕ МЕТОДА ЭЙКОНАЛА В НЕЛИНЕЙНОЙ ЭЛЕКТРОДИНАМИКЕ НА ОСНОВЕ ПРИНЦИПА ГЕОМЕТРИЗАЦИИ
}

\begin{abstract}
Выведено уравнение эйконала для электромагнитной волны, распространяющейся во внешнем электромагнитном поле по законам нелинейной электродинамики. На основе принципа геометризации Логунова определены метрические тензоры эффективных римановых пространств для электродинамики Борна-Инфельда, нелинейной электродинамики Гейзенберга-Эйлера и параметризованной постмаксвелловской электродинамики. Проанализированы основные свойства этих нелинейных электродинамик.
\end{abstract}

Ключевые слова: принцип геометризации, нелинейная электродинамика, уравнение эйконала.

\section{1. ВВЕДЕНИЕ}

В работах Анатолия Алексеевича Логунова по теории гравитационного поля [1], [2] был сформулирован принцип геометризации. Он означает, что “. . . описание движения вещества под действием гравитационного поля в псевдоевклидовом пространстве-времени физически тождественно описанию движения вещества в соответствующем эффективном римановом пространстве".

Идея А. А. Логунова об эффективном римановом пространстве оказалась очень плодотворной. Она применима не только к теории гравитации, но и к теории любого физического поля в псевдоевклидовом пространстве-времени, лагранжиан которого содержит нелинейные члены при высших производных, в том числе и к нелинейной электродинамике.

Однако нелинейная электродинамика имеет ряд отличий от теории гравитации. Основными из них являются следующие:

а) система уравнений нелинейной электродинамики содержит только первые производные от тензора электромагнитного поля $F_{i k}=-F_{k i}$, в то время как уравнения гравитации являются уравнениями второго порядка относительно тензора гравитационного поля $\varphi_{i k}=\varphi_{k i}$;

*МАТИ - Российский государственный технологический университет им. К. Э. Циолковского, Москва, Россия. E-mail: ivk@abiturcenter.ru 
б) при наличии гравитационного поля движение вещества и всех полей материи согласно принципу геометризации происходят в едином эффективном римановом пространстве, а получаемое из уравнений нелинейной электродинамики эффективное риманово пространство относится только к электромагнитному полю.

Поиск метрического тензора эффективного риманова пространства в нелинейной электродинамике происходит иначе, чем в теории гравитации. В работе [3] было проведено исследование нелинейно-электродинамических законов распространения электромагнитных волн в частном случае, когда в качестве внешнего электромагнитного поля рассматривалось поле магнитного диполя, причем расчеты проводились лишь с постмаксвелловской точностью.

Покажем, как реализуется принцип геометризации Логунова в общем случае нелинейной электродинамики, лагранжиан которой локально зависит от двух инвариантов электромагнитного поля $I_{2}=F_{i k} F^{k i}, I_{4}=F_{i k} F^{k n} F_{n m} F^{m i}$, и исследуем основные следствия этого принципа.

\section{2. СИСТЕМА УРАВНЕНИЙ НЕЛИНЕЙНОЙ ЭЛЕКТРОДИНАМИКИ}

В случае, когда лагранжиан $L$ является локальной функцией инвариантов $I_{2}$ и $I_{4}$, система уравнений нелинейной электродинамики вне источников имеет вид

$$
\begin{aligned}
& \frac{\partial}{\partial x^{k}}\left[Y_{2} F_{\cdot i}^{k \cdot}+2 Y_{4} F_{(3) \cdot i}^{k \cdot}\right]=0, \\
& \frac{\partial F_{n m}}{\partial x^{j}}+\frac{\partial F_{m j}}{\partial x^{n}}+\frac{\partial F_{j n}}{\partial x^{m}}=0,
\end{aligned}
$$

где $F_{(3)}^{k i}=F^{k n} F_{n m} F^{m i}$ - третья степень [4] тензора электромагнитного поля, $Y_{2}=$ $\partial L / \partial I_{2}, Y_{4}=\partial L / \partial I_{4}$. Здесь и далее точками обозначены вакансии для поднятия и опускания индексов.

Независимыми уравнениями системы (1) являются шесть уравнений, получаемых при подстановке индексов $i=n=\alpha, j=0, m=\beta$. Раскрывая производные в первом уравнении системы (1), получим

$$
\begin{gathered}
Y_{2} g^{k i} \frac{\partial F_{i l}}{\partial x^{k}}+2 F_{\cdot \alpha}^{k \cdot}\left[Y_{22} F^{n i} \frac{\partial F_{i n}}{\partial x^{k}}+2 Y_{24} F_{(3)}^{n i} \frac{\partial F_{i n}}{\partial x^{k}}\right]+ \\
+2 Y_{4}\left[F_{(2) \cdot \alpha}^{n \cdot} g^{k i} \frac{\partial F_{i n}}{\partial x^{k}}+F^{k n} F_{\cdot \alpha}^{m \cdot} \frac{\partial F_{n m}}{\partial x^{k}}+F_{(2)}^{k m} \frac{\partial F_{m \alpha}}{\partial x^{k}}\right]+ \\
+4 F_{(3) \cdot \alpha}^{k \cdot}\left[2 Y_{44} F_{(3)}^{n i} \frac{\partial F_{i n}}{\partial x^{k}}+Y_{24} F^{n i} \frac{\partial F_{i n}}{\partial x^{k}}\right]=0, \\
\frac{\partial F_{\alpha \beta}}{\partial x^{0}}+\frac{\partial F_{\beta 0}}{\partial x^{\alpha}}+\frac{\partial F_{0 \alpha}}{\partial x^{\beta}}=0
\end{gathered}
$$

где $Y_{22}=\partial Y_{2} / \partial I_{2}, Y_{24}=\partial Y_{2} / \partial I_{4}, Y_{44}=\partial Y_{4} / \partial I_{4}$.

В галлилеевых координатах псевдоевклидового пространства-времени тензор электромагнитного поля можно представить в интегральном виде:

$$
F_{n m}=\int d^{3} k \tilde{f}_{n m}(\mathbf{k}) \exp \{-i S(\mathbf{r}, t, \mathbf{k})\},
$$


где $S(\mathbf{r}, t, \mathbf{k})$ - эйконал.

Подставляя (3) в производные от тензора электромагнитного поля, входящие в уравнение (2), и комбинируя их, приведем систему (2) к виду

$$
\Pi_{\alpha}^{\nu} \tilde{f}_{0 \nu}=0
$$

где

$$
\begin{aligned}
\Pi_{\alpha}^{\nu}=[ & \left.Y_{2} R+2 Y_{4} P\right] \delta_{\alpha}^{\nu}+Y_{4} R F_{(2) \cdot \alpha}^{\nu \cdot}-\left[Y_{2} g^{k \nu}+2 Y_{4} F_{(2)}^{k \nu}\right] X_{k} X_{\alpha}+ \\
& +2\left[8 Y_{44} F_{(3) \cdot \alpha}^{k \cdot} F_{(3)}^{\nu n}-Y_{4} F_{(2) \alpha}^{n} g^{k \nu}-Y_{4} F^{k \nu} F_{\cdot \alpha}^{n \cdot}+2 Y_{22} F_{\cdot \alpha}^{k \cdot} F^{\nu n}+\right. \\
& \left.+4 Y_{24}\left(F_{\cdot \alpha}^{k \cdot} F_{(3)}^{\nu n}+F_{\cdot \alpha}^{k \cdot} F^{\nu n}\right)\right] X_{k} X_{n} .
\end{aligned}
$$

Здесь введены обозначения

$$
X_{n}=\frac{\partial S}{\partial x^{n}}, \quad P=F_{(2)}^{k n} X_{k} X_{n}, \quad R=X_{k} X_{n} g^{n k}
$$

Для существования нетривиальных решений уравнения (4) необходимо выполнение условия $\operatorname{det}\left\|\Pi_{\alpha}^{\nu}\right\|=0$. Согласно [4] это условие эквивалентно равенству

$$
2 \Pi_{(3)}-3 \Pi_{(2)} \Pi_{(1)}+\Pi_{(1)}^{3}=0,
$$

где $\Pi_{(1)}=\Pi_{\alpha}^{\alpha}, \Pi_{(2)}=\Pi_{\beta}^{\alpha} \Pi_{\alpha}^{\beta}, \Pi_{(3)}=\Pi_{\beta}^{\alpha} \Pi_{\mu}^{\beta} \Pi_{\alpha}^{\mu}-$ инварианты степеней тензора $\Pi_{\alpha}^{\nu}$ в трехмерном пространстве.

Однако если подставить выражение (5) в равенство (6), то в полученном соотношении трудно выделить инвариантные и неинвариантные части. Поэтому введем четырехмерное обобщение $Q_{m}^{n}$ трехмерного тензора $\Pi_{\alpha}^{\nu}$ в соответствии с равенством $Q_{\alpha}^{\nu}=\Pi_{\alpha}^{\nu}$.

В терминах $Q_{m}^{n}$ равенство (6) принимает вид

$$
2 Q_{(3) n}^{n}-3 Q_{(1) n}^{n} Q_{(2) m}^{m}+\left(Q_{m}^{n}\right)^{3}-6 Q_{(3) 0}^{0} Q_{n}^{n}+3\left[Q_{(2) n}^{n}-\left(Q_{n}^{n}\right)^{2}\right] Q_{0}^{0}=0 .
$$

Это равенство содержит как ковариантную часть, так и нековариантную. Поскольку оно должно выполняться во всех допустимых [1] системах отсчета псевдоевклидова пространства-времени, то для этого необходимо и достаточно, чтобы было выполнено равенство

$$
2 Q_{(3) n}^{k}-2 Q_{(2) n}^{k} Q_{m}^{m}-Q_{n}^{k}\left[Q_{(2) m}^{m}-\left(Q_{m}^{m}\right)^{2}\right]=0 .
$$

Подставляя четырехмерное обобщение тензора (5) в это равенство, получим

$$
P_{n}^{k}=\left[\delta_{n}^{k} R-\gamma^{k m} X_{n} X_{m}\right]\left[I_{2} Y_{4}+Y_{2}\right] f=0
$$


где введено обозначение

$$
\begin{aligned}
f=R^{2} & \left\{I_{2}^{4} Y_{4} Y_{44}+I_{2}^{4} Y_{44} Y_{22}-I_{2}^{4} Y_{24}^{2}+I_{2}^{3} Y_{2} Y_{44}-I_{2}^{2} Y_{4}^{2}-4 I_{2}^{2} I_{4} Y_{4} Y_{44}-\right. \\
& -I_{2}^{2} Y_{4} Y_{22}-4 I_{2}^{2} I_{4} Y_{44} Y_{22}+4 I_{2}^{2} I_{4} Y_{24}^{2}+2 I_{2}^{2} Y_{2} Y_{24}-I_{2} Y_{2} Y_{4}-2 I_{2} I_{4} Y_{2} Y_{44}+ \\
& \left.+2 I_{4} Y_{4}^{2}+4 I_{4}^{2} Y_{4} Y_{44}+2 I_{4} Y_{4} Y_{22}+4 I_{4}^{2} Y_{44} Y_{22}-4 I_{4}^{2} Y_{24}^{2}-4 I_{4} Y_{2} Y_{24}-Y_{2}^{2}\right\}+ \\
& +2 R P\left\{-I_{2}^{3} Y_{4} Y_{44}-2 I_{2}^{3} Y_{44} Y_{22}+2 I_{2}^{3} Y_{24}^{2}-I_{2}^{2} Y_{2} Y_{44}-I_{2} Y_{4}^{2}+2 I_{2} I_{4} Y_{4} Y_{44}+\right. \\
& \left.+4 I_{2} I_{4} Y_{44} Y_{22}-4 I_{2} I_{4} Y_{24}^{2}-4 I_{2} Y_{2} Y_{24}-3 Y_{2} Y_{4}-2 I_{4} Y_{2} Y_{44}-2 Y_{2} Y_{22}\right\}+ \\
& +8 P^{2}\left\{I_{2}^{2} Y_{22} Y_{44}-I_{2}^{2} Y_{24}^{2}-2 I_{2} Y_{4} Y_{24}-Y_{4}^{2}-2 I_{4} Y_{4} Y_{44}-\right. \\
& \left.-Y_{4} Y_{22}-2 I_{4} Y_{44} Y_{22}+2 I_{4} Y_{24}^{2}\right\} .
\end{aligned}
$$

Уравнение (7) содержит три сомножителя. Для того чтобы рассматриваемая нелинейная модель электродинамики в пределе слабого поля переходила в электродинамику Максвелла, в нуль должен обратиться последний сомножитель выражения (7): $f=0$. Именно это соотношение и представляет собой уравнение эйконала для произвольной нелинейной электродинамики вакуума, локально зависящей от инвариантов электромагнитного поля.

\section{3. ОПРЕДЕЛЕНИЕ МЕТРИЧЕСКОГО ТЕНЗОРА ЭФФЕКТИВНОГО РИМАНОВА ПРОСТРАНСТВА В НЕЛИНЕЙНОЙ ЭЛЕКТРОДИНАМИКЕ}

Рассмотрим вид уравнения эйконала $f=0$ в различных моделях нелинейной электродинамики вакуума и определим, используя принцип геометризации Логунова, метрический тензор эффективного риманова пространства для каждой из них.

В современной научной литературе рассматриваются три модели нелинейной электродинамики вакуума: электродинамика Борна-Инфельда [5], нелинейная электродинамика Гейзенберга-Эйлера [6] и параметризованная постмаксвелловская электродинамика [7]. В нелинейной электродинамике Борна-Инфельда функция $L$ имеет вид

$$
L=\frac{4}{a^{2}}\left\{1-\sqrt{1-\frac{a^{2}}{2} I_{2}-\frac{a^{4}}{4} I_{4}+\frac{a^{4}}{8} I_{2}^{2}}\right\},
$$

где $a$ - постоянная, для которой известна лишь оценка снизу [5]: $a^{2}>1.2 \cdot 10^{-32} \Gamma^{-2}$.

В нелинейной электродинамике Гейзенберга-Эйлера лагранжиан обычно записывают в приближении слабого поля:

$$
L=\frac{1}{16 \pi}\left\{I_{2}+\frac{\alpha}{180 \pi B_{q}^{2}}\left(14 I_{4}-5 I_{2}^{2}\right)\right\},
$$

где $\alpha=e^{2} / \hbar c \approx 1 / 137$ - постоянная тонкой структуры, а $B_{q}=m^{2} c^{2} / e \hbar \approx 4.41 \cdot 10^{13} \Gamma \mathrm{c}$ - характерная квантовоэлектродинамическая индукция.

И наконец, в параметризованной постмаксвелловской электродинамике вакуума лагранжиан содержит два свободных параметра $\eta_{1}$ и $\eta_{2}$ :

$$
L=\frac{1}{32 \pi}\left\{2 I_{2}+\xi\left[\left(\eta_{1}-2 \eta_{2}\right) I_{2}^{2}+4 \eta_{2} I_{4}\right]\right\},
$$


где $\xi=1 / B_{q}^{2}$.

Величина безразмерных постмаксвелловских параметров $\eta_{1}$ и $\eta_{2}$ зависит от выбора модели нелинейной электродинамики вакуума: в нелинейной электродинамике Гейзенберга-Эйлера эти параметры имеют значения $\eta_{1}=\alpha /(45 \pi)=5.1 \cdot 10^{-5}$, $\eta_{2}=7 \alpha /(180 \pi)=9.0 \cdot 10^{-5}$, а в теории Борна-Инфельда они выражаются через постоянную $a^{2}$ следующим образом: $\eta_{1}=\eta_{2}=a^{2} B_{q}^{2} / 4$.

Подставляя лагранжиан (9) нелинейной электродинамики Борна-Инфельда в соотношение (8) и потребовав $f=0$, получим

$$
\left\{\left[\gamma^{k m}\left[1-\frac{a^{2} I_{2}}{2}\right]+a^{2} F_{(2)}^{k m}\right] \frac{\partial S}{\partial x^{k}} \frac{\partial S}{\partial x^{m}}\right\}^{2}=0,
$$

где $\gamma^{k m}$ - метрический тензор псевдоевклидова пространства-времени.

В нелинейной электродинамике Гейзенберга-Эйлера с лагранжианом (10) уравнение $f=0$ принимает вид

$$
\left\{\left[\gamma^{k m}+\frac{4 \alpha}{45 \pi B_{q}^{2}} F^{k n} F_{n \cdot}^{\cdot m}\right] \frac{\partial S}{\partial x^{k}} \frac{\partial S}{\partial x^{m}}\right\}\left\{\left[\gamma^{i j}+\frac{7 \alpha}{45 \pi B_{q}^{2}} F^{i p} F_{p \cdot}^{\cdot j}\right] \frac{\partial S}{\partial x^{i}} \frac{\partial S}{\partial x^{j}}\right\}=0 .
$$

Совершенно аналогично, подставляя лагранжиан (11) параметризованной постмаксвелловской электродинамики вакуума в соотношение (8) и потребовав $f=0$, получим

$$
\left\{\left[\gamma^{k m}+4 \xi \eta_{1} F^{k n} F_{n \cdot}^{\cdot m}\right] \frac{\partial S}{\partial x^{k}} \frac{\partial S}{\partial x^{m}}\right\}\left\{\left[\gamma^{i j}+4 \xi \eta_{2} F^{i p} F_{p^{\cdot}}^{\cdot j}\right] \frac{\partial S}{\partial x^{i}} \frac{\partial S}{\partial x^{j}}\right\}=0 .
$$

Из уравнения (12) следует, что в электродинамике Борна-Инфельда электромагнитные волны распространяются независимо от их поляризации, и метрический тензор эффективного риманова пространства в этом случае имеет вид

$$
g^{k m}=\gamma^{k m}\left[1-\frac{a^{2} I_{2}}{2}\right]+a^{2} F_{(2)}^{k m} .
$$

Используя выражение (15) и формулы тензорной алгебры [4], мы без труда можем найти ковариантные компоненты метрического тензора эффективного риманова пространства и его определитель в электродинамике Борна-Инфельда:

$$
g_{i k}=\frac{8\left[\gamma_{i k}-a^{2} F_{i k}^{(2)}\right]}{8-4 a^{2} I_{2}-2 a^{4} I_{4}+a^{4} I_{2}^{2}}, \quad g=\gamma\left[1-\frac{a^{2}}{2} I_{2}-\frac{a^{4}}{4} I_{4}+\frac{a^{4}}{8} I_{2}^{2}\right]^{2},
$$

где $\gamma$ - определитель метрического тензора $\gamma_{i k}$ псевдоевклидова пространства-времени.

Из выражений (13) и (14) следует, что в электродинамике Гейзенберга-Эйлера и в параметризованной постмаксвелловской электродинамике при $\eta_{1} \neq \eta_{2}$ электромагнитные волны во внешних электромагнитных полях распространяются в виде двух нормальных волн со взаимно ортогональной поляризацией. Первая из них распространяется в эффективном римановом пространстве с метрическим тензором $g_{(1)}^{k m}$, а вторая - в пространстве с метрическим тензором $g_{(2)}^{k m}$, где

$$
g_{(1,2)}^{k m}=\gamma^{k m}+4 \xi \eta_{1,2} F^{k n} F_{n \cdot}^{\cdot m} .
$$


Установив метрический тензор эффективного риманова пространства для той или иной нелинейной электродинамики, мы можем проводить дальнейшие исследования законов распространения фотонов во внешних электромагнитных полях, решая уравнения для изотропных геодезических в этом эффективном римановом пространстве.

\section{4. ЗАКЛЮЧЕНИЕ}

Итак, электродинамика Гейзенберга-Эйлера и параметризованная постмаксвелловская электродинамика при $\eta_{1} \neq \eta_{2}$ предсказывают существование двойного лучепреломления электромагнитных волн во внешних электромагнитных полях, аналогичное двойному лучепреломлению в кристаллических средах. Поэтому согласно этим теориям электромагнитные волны во внешнем электромагнитном поле должны распространяться в виде двух ортогонально поляризованных нормальных волн вдоль различных лучей и с различной скоростью.

В нелинейной электродинамике Борна-Инфельда воздействие внешних электромагнитных полей на электромагнитные волны осуществляется независимо от их поляризации. На этих различиях в предсказаниях различных моделей нелинейной электродинамики и основаны эксперименты, которые призваны дать ответ на вопрос, какая из этих моделей наиболее адекватна природе.

Благодарности. Настоящая работа выполнена при частичной поддержке Программы поддержки ведущих научных школ (грант № НШ-4476.2006.2) и РФФИ (грант № 06-02-16514).

\section{Список литературы}

[1] А.А. Логунов, Лекиии по теории относительности и гравитации, Наука, М., 1987.

[2] А. А. Логунов, Теория гравитационного поля, Наука, М., 2000; А. А. Логунов, М. А. Мествиришвили, Основы релятивистской теории гравитации, МГУ, М., 1983.

[3] В. И. Денисов, ТМФ, 132:2 (2002), 211.

[4] И.П. Денисова, Введение в тензорное исчисление и его приложения, УНЦ ДО, М., 2004.

[5] M. Born, L. Infeld, Proc. Roy. Soc. A, 144 (1934), 425.

[6] W. Heisenberg, H. Euler, Z. Phys., 26 (1936), 714.

[7] I. P. Denisova, I. V. Krivchenkov, P. A. Vshivtseva, A. A. Zubrilo, Gen. Relat. Gravit., 36:4 (2004), 889. 